\title{
New Absolute Contrast for pulsed thermography
}

\author{
by M. Pilla ${ }^{1}$, M. Klein ${ }^{2}$, X. Maldague ${ }^{3}$, A. Salerno ${ }^{1}$ \\ (1) Politecnico di Milano, Dip. di Energetica, P.zza L. Da Vinci 32, 20133 Milano, Italy. \\ (2) Ericsson Datacom Inc, 70 Castilian Dr, Santa Barbara, CA 93117, USA. \\ (3) Université Laval, Quebec City (Quebec) G1K 7P4, Canada [maldagx@gel.ulaval.ca]
}

\begin{abstract}
In this paper, a new absolute thermal contrast method is proposed for pulsed infrared thermography. It is based on the computations of reconstructed defect-free images so that no a priori knowledge of a sound area on the sample is necessary. Moreover, a correction is applied to take into account possible delays in the acquisition time. Results are presented both on Plexiglas ${ }^{\mathrm{TM}}$ and graphite-epoxy specimens. Comparisons with Pulsed Phase Thermography phase images are also presented along with a discussion on the advantages of the proposed method.
\end{abstract}

\section{Introduction}

Pulsed thermography (PT) is a common thermal stimulation approach in active infrared thermography (AIRT) [1]. Among established thermogram processing techniques, thermal contrast computations are convenient in order to enhance subsurface defect visibility, and also to enable some quantitative extractions such as defect depth, size and thermal properties. Thermal contrast definitions imply the knowledge of a sound area within the infrared camera field of view [1]. This is obviously a severe limitation since it requires a priori information not always available (after all, the purpose of AIRT is precisely to delineate sound areas!). Moreover, in these thermal contrast computations, the temperature of the sound area is generally assumed uniform all over the specimen and thus reduced to a scalar value. Of course this is untrue if the energy deposited over the specimen is not uniform nor if the thermal properties of the material (ex: thermal effusivity) change over the specimen. In all these cases, traditional contrast computations lead to inaccuracies [2].

In this paper, a new technique is proposed to overcome these difficulties in order to obtain a reliable thermal contrast. First of all a method is proposed to compute the local temperature of a sound area $T s_{[i, j]}(t)$ by mean of an heat transfer model (semi-infinite body assumption) and without a priori knowledge of sound area locations (here [i,j] represents location within the field of view, $T$ the temperature, $t$ the time and subscript $s$ stands for sound area). Second, the inaccuracy of the acquisition time is also taken into account and corrected.

\section{Reconstructed sound area temperature images}

In $\mathrm{PT}$, the specimen is submitted to a thermal pulse at $t=0$, the thermal pulse last up to $t_{f}$ and a thermogram sequence is recorded. Once temperature is recorded over the surface with the infrared (IR) camera, it takes a moment before the defects manifest themselves due to the travelling time of the thermal waves within the specimen [1]. Let assume $t$ ' is a time between $t_{f}$ and the time at which the first temperature spot related to subsurface defects appears. In this case, we have $T s_{[i, j]}\left(t^{\prime}\right)=T_{[i, j]}\left(t^{\prime}\right)$. Next we compute $T s_{[i, j]}$ for all the rest of the temporal sequence. Assuming a Dirac pulse applied to a semi-infinite body, the 1-dimensional Fourier equation is solved as ( $z$ is the depth variable, $z=0$ corresponds to the surface, $Q$ is the injected energy at the surface, $e$ is the thermal effusivity of the sample and $\Delta T$ is the temperature increase from $t=0)[3$, see also the discussion in 4]: 


$$
\Delta T_{\text {semi-inf inite-body }}(z=0, t)=\frac{Q}{e \sqrt{\pi t}}
$$

As it is well known, the solution provided by eq. (1) diverges as time elapses and also as plate thickness enlarges with respect to the non-semi-infinite case [4]. For instance, in case of Plexiglas $^{\mathrm{TM}}$, it was found the error obtained with eq. (1) is less than $2 \%$ for observation time of less than $2 \mathrm{~s}$ and plate thickness less than $1 \mathrm{~mm}$. In case of an $\mathrm{Al}$ specimen, error is less than $20 \%$ for time of $0.02 \mathrm{~s}$ and $2 \mathrm{~mm}$ plate thickness [5]. Nevertheless, eq. (1) is a good approximation.

At time $t^{\prime}$, the temperature of the sound area $T s_{[i, j]}\left(t^{\prime}\right)$ is then given by:

$$
\Delta T s_{[i, j]}\left(t^{\prime}\right)=\Delta T_{[i, j]}\left(t^{\prime}\right)=\frac{Q_{[i, j]}}{e_{[i, j]} \sqrt{\pi t^{\prime}}}
$$

Assuming injected energy over the specimen is changing relatively smoothly, eq. (1) stands and allows to extract $Q / e$ locally:

$$
\frac{Q_{[i, j]}}{e_{[i, j]}}=\sqrt{\pi t^{\prime}} \cdot \Delta T_{[i, j]}\left(t^{\prime}\right)
$$

From eq. (3), the temperature of the sound area can be defined locally as a function of $t$ :

$$
\Delta T s_{[i, j]}(t)=\frac{Q_{[i, j]}}{e_{[i, j]} \sqrt{\pi t}}=\frac{\sqrt{\pi t^{\prime}}}{\sqrt{\pi t}} \cdot \Delta T_{[i, j]}\left(t^{\prime}\right)=\sqrt{\frac{t^{\prime}}{t}} \cdot \Delta T_{l, J}\left(t^{\prime}\right)
$$

Interestingly, performing eq. (4) computations over all the surface (all locations $[i, j]$ ) and for the whole temporal sequence) reconstructs the "ideal" defect-free thermogram sequence.

\section{New Absolute Contrast (NAC)}

Now lets introduce the well known absolute thermal contrast definition $C^{a c}[1]$ :

$$
C_{[i, j]}^{a c}(t)=T_{[i, j]}(t)-T s(t)
$$

The new proposed thermal contrast definition is first generalized over all thermogram locations:

$$
C_{[i, j]}^{a c}(t)=T_{[i, j]}(t)-T s_{[i, j]}(t)
$$

We can then rewrite eq. (6) in terms of $\Delta T$ as follows:

$$
C_{[i, j]}^{a c}(t)=\left\{T_{[i, j]}(t)-T_{[i, j]}(0-)\right\}-\left\{T s_{[i, j]}(t)-T s_{[i, j]}(0-)\right\}
$$

where $T_{[i, j]}\left(0_{-}\right)$is the absolute temperature of the specimen at location [i,j] before heating (i.e. at $t=0-$ ), this is generally referred to as the cold image. Obviously we also have $T_{[i, j]}(0-)=T s_{[i, j]}(0-)$ since no defects are present at $t=0$ - and both cancel out in eq. (7) so that from eq. (5) we can write:

$$
C_{[i, j]}^{a c}(t)=\Delta T_{[i, j]}(t)-\Delta T s(t)
$$

Finally, the New Absolute Contrast (NAC) is obtained as:

$$
C_{[i, j]}^{n a c}(t)=\Delta T_{[i, j]}(t)-\sqrt{\frac{t^{\prime}}{t}} \Delta T_{i, j}\left(t^{\prime}\right)
$$

\section{Time considerations}

Since eq. (1) as the form $\Delta T=c^{\circ} \mathrm{t}^{\mathrm{a}}$, in logarithmic space it can be re-written as (see $[4,7]$ for instance) $\log (\Delta T)=a \cdot \log (t)+b$ where $a$ and $b$ are constant for a given location 
$[i$,$] on the specimen surface and with a=-1 / 2$ and $b=\log (Q /(e \sqrt{ } \pi))$. As it is well known, the good thing with such representation is that the logarithmic time behavior of a defectfree region is a line with $-1 / 2$ slope.

In eq. (9) time $t$ and $t^{\prime}$ are referenced to $t_{f}$. Any error $t_{\varepsilon}$ on $t_{f}$ will affect NAC computations, especially when a logarithmic space is chosen. For instance Figures 1a and $1 \mathrm{~b}$ show such effect for positive and negative values of $t_{\varepsilon}$. In the figure the curve $\Delta T$ vs. $(t+)$ is plotted (case of a plastic plate, no defect). The straight corresponds to the ideal $1 / 2$ slope $T s(t)$ case. Obviously, the actual data does not match the ideal case and such error $t_{\varepsilon}$ will affect further NAC computations.
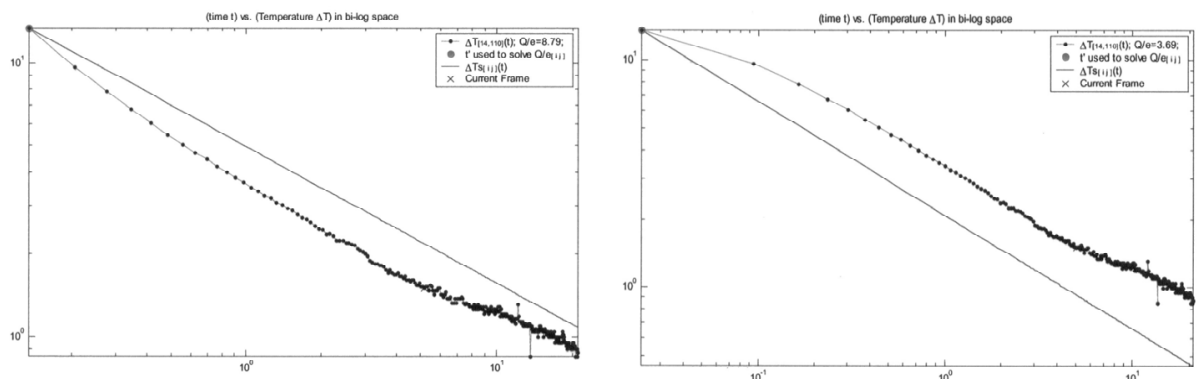

(a)

(c)

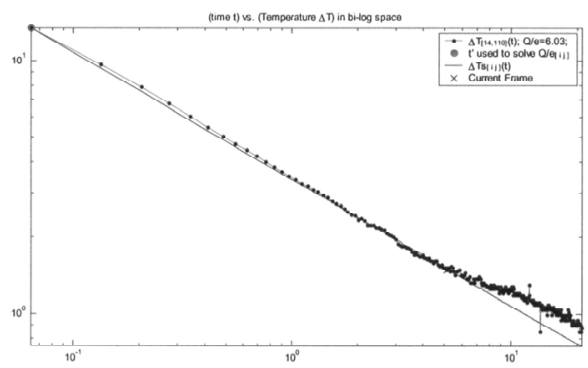

(b)

Fig. 1. (a) Small positive error on time $t_{\varepsilon}$, (b) Small negative error on time $t_{\varepsilon}$,

(c) Error compensated

The solution to this is to find the error $t_{\varepsilon}$ and then to compensate it by computing:

$$
C_{[i, j]}^{n a c}\left(t-t_{\varepsilon}, t^{\prime}-t_{\varepsilon}\right) \text { instead of } C_{[i, j]}^{n a c}\left(t, t^{\prime}\right)
$$

Normally, such procedure should lead to a curve with a linear behavior, at least at the earlier times (due to unaccounted thermal lateral flow, thermal losses), see for instance Figure 1-c. Such adjustment is made dynamically thanks to a dedicated computer program by modifying $t_{\varepsilon}$ so that both lines coincide together [10]. Interestingly, in [11] another method is shown to deal with a finite pulsed width in case the pulse is not fast enough.

\section{Experimental results}

\subsection{Plexiglas ${ }^{\mathrm{TM}}$ plate}

The $4 \mathrm{~mm}$-thick plate contains 6 flat-bottom holes of depth $1,1.5,2,2.5,3,3.5 \mathrm{~mm}$, all with $10 \mathrm{~mm}$ diameter. This academic specimen was flashed heated for $15 \mathrm{~ms}$ (with two flashes of $6.4 \mathrm{KJ}$ of electrical energy per flash) and 200 thermograms were recorded (from $t=0.1$ to $t=20 \mathrm{~s}$ ). The experimental apparatus is described elsewhere [8]. Figure 2-a shows $\Delta T$ vs. time for three locations over the specimen (first 2 curves over defect and last 
one over sound area). Error $t_{\varepsilon}$ was corrected as explained in previous section. The computed - eq. (3) - values Q/e are respectively 7.97, 6.72 and 6.05 confirming the nonuniform heating visible on the raw thermogram of Figure 2-b. The dots at $t=0.9 \mathrm{~s}$ correspond to $t^{\prime}$. The straights represents:

$$
\Delta T s_{[i, j]}(t)=\sqrt{\frac{t^{\prime}}{t}} \cdot \Delta T_{I, J}\left(t^{\prime}\right)
$$

and the dotted curves $\Delta T_{[i, j]}(t)$. The NAC corresponds, respectively for the three cases, to the difference between the dotted curve and the straight as in eq. (9). Figure 3-a shows the NAC at $t=6.4 \mathrm{~s}$. For reference, the phase image at frequency $f=0.5 \mathrm{~Hz}$ in Pulsed Phase Thermography (PPT) [9] is provided in Figure 3-b as well. As it is seen both images are similar with defects showing over a relatively flat background (compare with raw thermogram of Figure 2-b). The absolute contrast image is not provided as it is similar to Figure 2-a (since a constant value is simply subtracted from each image - eq. (5) - this does not modify the relationship between defects and background).

(a)

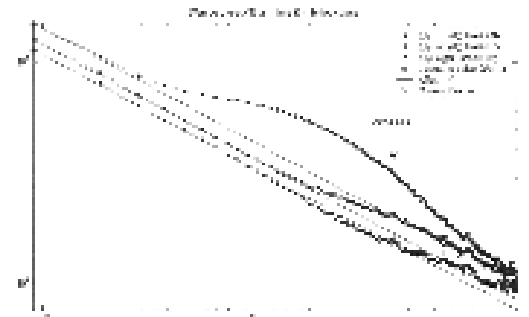

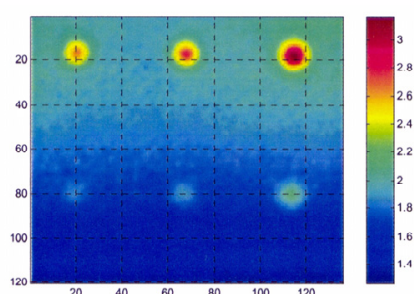

(b)

Fig. 2. Plexiglas specimen: (a) $\Delta T_{[i, j]}(t)$ and $\Delta T s_{[i, j]}(t)$ plotted for 3 locations over (b), black dots represent the acquisition time for each thermogram, crosses indicate the acquisition time of (b). (b) Raw thermogram at $t=6.40 \mathrm{~s}$

$\mathrm{t}=6.40 \mathrm{~s}$

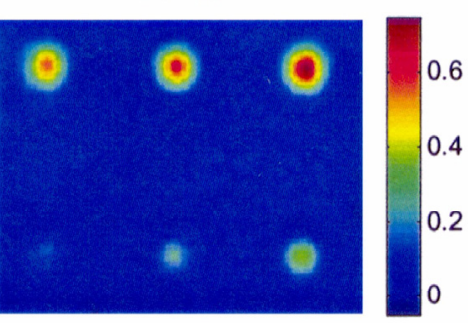

$f=0.5 \mathrm{~Hz}$

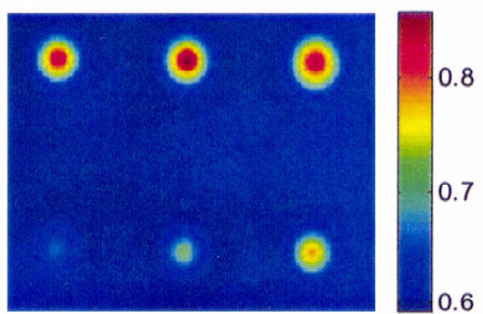

Fig. 3. (a) New absolute contrast image at $t=6.40 \mathrm{~s}$, (b) PPT phase image at $f=0.5 \mathrm{~Hz}$ (plastic specimen)

\subsection{Graphite-epoxy plate}

The next specimen is a graphite-epoxy plate of 5 plies. Simulated defects as a Teflon insert and a thin void were embedded at manufacturing stage. Fifty images were acquired from $0.55 \mathrm{~s}$ to $1.53 \mathrm{~s}$. The same experimental apparatus as in previous section was used. Figure 4 shows three raw thermograms recorded at different times. Figure 5 shows the NAC for the same three moments. Both defects are clearly seen as also a possible delamination or epoxy-richer zone on the right side of the specimen. For reference, Figure 6 shows the phase image at frequency $f=1 \mathrm{~Hz}$ in PPT. In this case, the NAC provides better results. 
$t=0.57 s$

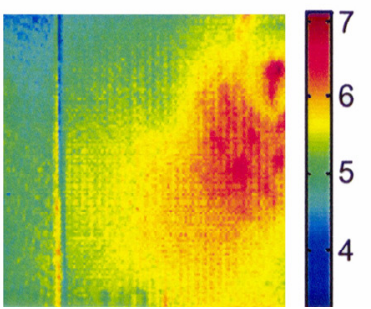

$t=1.17 s$

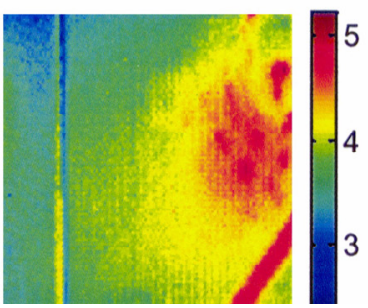

$t=1.53$

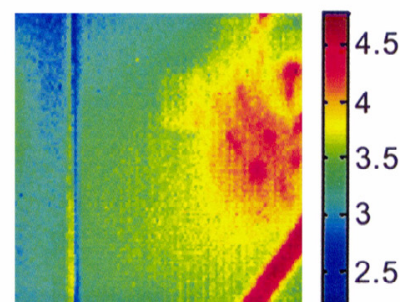

Fig. 4. Raw thermogram (graphite-epoxy specimen) at $t=0.57,1.17,1.53 \mathrm{~s}$

$t=0.57 \mathrm{~s}$

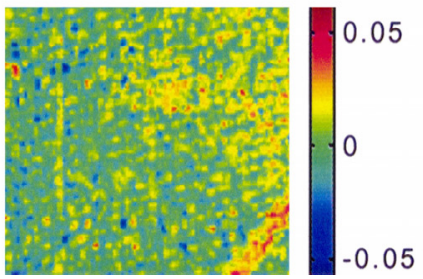

$t=1.17 \mathrm{~s}$

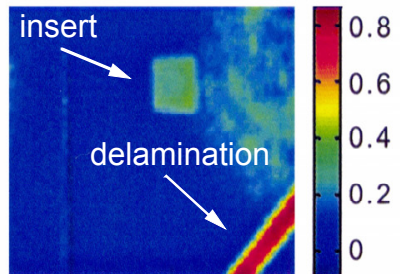

$t=1.53$

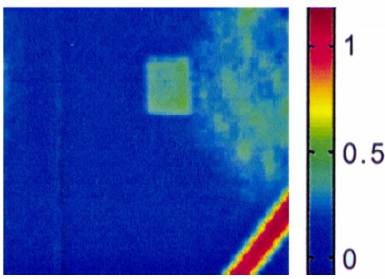

Fig. 5. New absolute contrast image (graphite-epoxy specimen) at $t=0.57,1.17,1.53 \mathrm{~s}$

$$
\mathrm{f}=1 \mathrm{~Hz}
$$

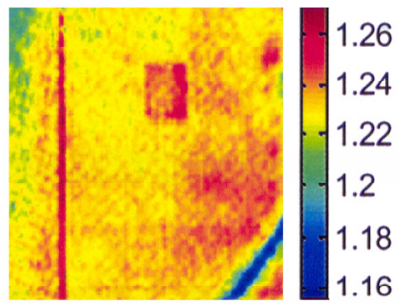

Fig. 6. PPT phase image (graphite-epoxy specimen) at $f=1 \mathrm{~Hz}$

\section{Discussion}

As seen, the NAC is a novel processing technique that improves the signal to noise ratio using a simple and fast - no complex computations in eq. (9) - algorithm by computing locally the sound area values $T s$. In fact, it brings the following advantages to PT:

- For a given time $t$, only two thermograms (at $t^{\prime}$ and at $t$ of interest) are needed to compute the NAC while for instance the PPT approach requires many more (100-150).

- In quantitative studies (ex: to extract defect depth), one approach consists to compute the maximum contrast. Since this is computed precisely with the NAC (ex: the local injected energy is taken into account), higher reliability is achieved.

- Since the background is made "flat", defects are segmented easily, especially in case of non uniform heating or local emissivity variations.

- Ts computed in the NAC can be applied to other contrast definitions [1] as well.

Thus, the known Absolute thermal Contrast is strongly enhanced thanks to a better definition of the sound area Ts. The Absolute Contrast is only taken as an example to show the interests about computing this new $T s_{[i, j]}\left(t^{\prime}\right)$. The local sound area $T s_{[i, j]}\left(t^{\prime}\right)$ can be applied to any equation involving a sound area Ts, especially it can enhance most of type 
of contrasts and not only the absolute contrast. In 1987, [12-13] found out a powerful way to show defects they called it the "Normalized Apparent Effusivity' (NAE). For instance, an advantage of the NAE was its ability to maintain a constant background from image to image. Interestingly, the local $T s_{[i, j]}\left(t^{\prime}\right)$ helps explaining the NAE. In fact, the NAE is the inverse of the Running Contrast using $T s_{[i, j]}\left(t^{\prime}\right)$. From this point, the NAE can be seen as a particular case of a general method presented in this article.

\section{Conclusion}

In this paper a new absolute contrast (NAC) procedure is derived and proposed based on the reconstruction of the temperature of the sound area. It was also shown how important is to adjust uncertainties in the acquisition time. Results were presented on two specimens with comparisons with PPT. Due to its distinctive advantages, it is believed the interests of the local $\mathrm{Ts}_{[\mathrm{i}, \mathrm{j}]}\left(\mathrm{t}^{\prime}\right)$ will be well received by the AIRT, PT community.

\section{Acknowledgement}

This work is supported by the Natural Sciences and Engineering Research Council of Canada. Agusta helicopters is thanked for the supply of specimens.

\section{REFERENCES}

[1] MALDAGUE (X.)., Theory and Practice of Infrared Thermography for Nondestructive Evaluation, New York, John Wiley pub., 2001, $684 \mathrm{p}$

[2] GRINZATO E., MARINETTI S., BISON P.G., "NDE of Porosity in CFRP by multiple thermographic techniques", Proc. SPIE: Thermosense XXIII, 4710, 2002, 588-598 p

[3] CARLSLAW H.S., Jaeger J.C., Conduction of Heat in Solids, Oxford University Press, 2nd edition, 1959

[4] BALAGEAS D.L., DÉOM A. A., BOSCHER D. M., "Characterization and Nondestructive Testing of Carbon-Epoxy Composites by a Pulsed Photothermal Method", Materials Evaluation, 45 (April), 461-465.

[5] ALMOND D.P., SAINTEY M.B., LAU S. K., "Edge-effects and defect sizing by transient thermography", QIRT 1994, 1994, 247-252 p

[6] Same authors, "Considerations on new techniques to enhance the contrast" (text in preparation)

[7] SHEPARD S.M., "Advances in Pulsed Thermography" Proc. SPIE: Thermosense XXIII, 4360, 2001, 511-515 $p$

[8] DARABI A., MALDAGUE X., "Neural Networks Based Detection and Depth Estimation in TNDE," Nondestructive Testing and Evaluation International, 35 [3], 2002, 165-175 p

[9] MALDAGUE X., MARINETTI S., "Pulsed Phase Infrared Thermography," J. Appl. Phys, 79 1996, 2694-2698 $p$

[10] IR-VIEW, computer program in MATLAB code, Université Laval, 2002.

[11] AZUMI T. and TAKAHASHI Y., Rev. Sc. Instr., vol. 52, pp 1411-1413 (1981).

[12] BOSCHER D.M., BALAGEAS D.L., DEOM A.A. and GARDETTE G., "Non destructive evaluation of carbon expoxy laminates using transient infrared thermography ", Proceedings 16 th NDE Symposium, San Antonio (TX), USA, April 21-23, 1987.

[13] DEOM A., BOSCHER D., and BALAGEAS D.L., "Pulsed photothermal non destructive testing. Application to carbon expoxy laminates", Review of Progress in Quantitative Non Destructive Evaluation, vol. 9A, p. 525-531, 1990. 